\title{
Control of Aerial Manipulation Vehicle in Operational Space
}

\author{
Somasundar Kannan, Serket Quintanar Guzman, Jan Dentler, \\ Miguel A. Olivares-Mendez and Holger Voos \\ SnT- University of Luxembourg, \\ 6 rue Richard Coudenhove-Kalergi, Kirchberg \\ L-1359 Luxembourg \\ Email: somasundar.kannan@uni.lu
}

\begin{abstract}
Operational Space Control of an Aerial Manipulation Vehicle is discussed here. The Aerial Manipulation Vehicle has a highly coupled dynamics due to the interaction between the Quadrotor and the attached manipulator. The nonlinear coupling introduces disturbances on the quadrotor which hinders precise control. A control solution in the operational space is considered where the End-Effector has to reach a final position starting from an initial hovering position. A hierarchical control approach is implemented where the outermost layer consist of Closed Loop Inverse Kinematics algorithm followed by position and attitude control loop for the quadrotor. The robotic arm and the quadrotor are controlled by different combinations of PID control methods. The proposed method is successfully tested through simulations for position control of the Aerial Manipulator.

Keywords-Aerial Manipulator, Unmanned Aerial Vehicle (UAV), Robotic Arm, Autonomous Vehicle.
\end{abstract}

\section{INTRODUCTION}

The developments in the active tasking of Unmanned Aerial Vehicles (UAVs) has increased their employability by enhanced interaction with the environment. The active tasking includes applications such as grasping, manipulation, transportation etc. This has also lead to attention towards the problem of Aerial Manipulation, that is to actively manipulate an object using an aerial vehicle. The different applications of Aerial Manipulation have lead to a set of challenges to be pursued.

Here we will briefly discuss the problems that have been actively researched in the recent years. An experimental study and stability analysis was performed on a helicopter with load disturbances due to gripped object in [1]. A ducted fan was modelled and controlled in [2] to understand the interaction with the environment.

The problem of Cartesian Impedence control and redundancy was studied in [3] and [4] using Euler-Lagrange formulation. The Newton-Euler method had been used in [5] and [6] to model and control a quadrotor attached with manipulator. Model Reference Adaptive Control was used in [7] to stabilize a quadrotor with multi degree of freedom manipulator, where mainly the rigid body dynamics of the quadrotor were considered.

In [8] a simple gripper was attached to the fuselage of a helicopter and a vision based sensor was used to control the manipulator. In [9] experiments were performed on a quadrotor with a gripper and an IR camera was used as a sensor to detect and grip an object with LED attached on it.

In [10] the problem of load transportation was studied. Here dynamic programming was used to generate optimal trajectory to perform a swing free manoeuvring. Additionally an adaptive control was used to tackle the changes in center of gravity. In [11], [12] the problem due to shift in center of mass during manipulation of objects is handled using adaptive control. Here an adaptive position control was used while the inner loop consist of PD roll-pitch control with center of mass estimation.

In [13] the problem of disturbance caused by manipulator with payload is tackled mainly by adaptation of the outer position loop. In [14] the development of an Aerial Manipulation system where the complete non-linearity of the quadrotor is considered along with 2-link manipulator dynamics based on Recursive Newton Euler (RNE) formulation.

In [15] a hybrid image and position based visual servoing based on hierarchical task- composition was considered to control an aerial vehicle equipped with a six degree of freedom robotic arm. In [16] a 2 degree of freedom manipulator was controlled using an adaptive sliding mode control. In [17] simulation studies were performed to control a quadrotor with manipulator considering operational space control. Similarly in [18] adaptive control was discussed to control an Aerial vehicle with manipulator having six degree of freedom identical to [17].

In this paper we consider the problem of Aerial Vehicle equipped with a 2 degree of freedom robotic arm. An operational space control task has to be achieved using classical hierarchical control structure. Compared to the previous problems in [17] and [18] the quadrotor equipped with a 2 DOF robotic arm used in this paper has relatively lesser degrees of freedom to attain the required task in operational space .The paper is structured as follows. First the modelling is briefly discussed which includes the kinematics and dynamic modelling of the composite system of quadrotor and manipulator with 2 DOF. This leads us to the control discussion involving Closed Loop Inverse Kinematics, position and attitude control of the quadrtor and control of manipulator. Finally simulation studies are performed to analyse the control method. 


\section{Modelling}

Here we will briefly discuss the kinematics and dynamics of the Aerial Manipulation Vehicle. The discussion is based on [17]. The design of the Aerial Manipulation Vehicle can be seen in Figure-1 and the 2 degree of freedom manipulator can be seen in Figure-2.

\section{A. Kinematics}

Let us denote the vehicle body-fixed frame as $\mathcal{B}$ fixed to the center of mass of the quadrotor. The position of $\mathcal{B}$ with respect to the world fixed inertial frame $\mathcal{I}$ is given by the vector $p_{b}=$ $\left[\begin{array}{lll}x & y & z\end{array}\right]$. The orientation of $\mathcal{B}$ with respect to $\mathcal{I}$ is give by the rotation matrix $R_{b}\left(\varphi_{b}\right)$. Here we define $\varphi_{b}=\left[\begin{array}{ll}\psi & \phi\end{array}\right]$ as the Yaw-Pitch-Roll angles. Let us define $\mathcal{E}$ as the frame attached to the end-effector of the manipulator. The position of $\mathcal{E}$ with respect to $\mathcal{I}$ is given by [17]

$$
p_{e}=p_{b}+R_{b} p_{e b}^{b},
$$

where $p_{e b}^{b}$ describes the position of frame $\mathcal{E}$ with to frame $\mathcal{B}$. Similarly the velocity $\dot{p}_{e}$ of $\mathcal{E}$ in the frame $\mathcal{I}$ is given by [17]

$$
\dot{p}_{e}=\dot{p}_{b}-S\left(R_{b} p_{e b}^{b}\right) \omega_{b}+R_{b} \dot{p}_{e b}^{b}
$$

where $S(\cdot)$ is a skew symmetric matrix operator.The orientation of $\mathcal{E}$ can be described by the rotation matrix [17]

$$
R_{e}=R_{b} R_{e}^{b},
$$

where $R_{e}^{b}$ describes the orientation of $\mathcal{E}$ with respect to $\mathcal{B}$. Let $q$ be the joint coordinates of the manipulator. Then $p_{e b}^{b}(q)$ and $R_{e}^{b}(q)$ are described by the direct kinematics algorithm of the classic fixed base manipulator with respect to its base frame. The generalized velocity of the end-effector with respect to $\mathcal{B}$ can be obtained in-terms of joint velocities $\dot{q}$ as given below [17]

$$
v_{e b}^{b}=J_{e b}^{b}(q) \dot{q}
$$

where $J_{e b}^{b}$ is the manipulator Jacobian. The generalized endeffector velocity in the frame $\mathcal{I}$ can be give as [17]

$$
v_{e}=J_{b}\left(q, R_{b}\right) v_{b}+J_{e b}\left(q, R_{b}\right) \dot{q}
$$

where $v_{b}=\left[\begin{array}{ll}\dot{p}_{b}^{T} & \omega_{b}^{T}\end{array}\right]$ and we have

$$
J_{b}=\left[\begin{array}{cc}
I_{3} & -S\left(R_{b} p_{e b}^{b}\right) \\
O_{3} & I_{3}
\end{array}\right], J_{e b}=\left[\begin{array}{cc}
R_{b} & O_{3} \\
O_{3} & R_{b}
\end{array}\right] J_{e b}^{b},
$$

where $I_{3}$ and $O_{3}$ are identity and null matrices respectively. We can also express $v_{e}$ as follows [17]

$$
v_{e}=J_{b}\left(q, R_{b}\right) \dot{x}_{b}+J_{e b}\left(q, R_{b}\right) \dot{q},
$$

with

$$
x_{b}=\left[\begin{array}{l}
p_{b} \\
\varphi_{b}
\end{array}\right], T_{A}\left(\varphi_{b}\right)=\left[\begin{array}{cc}
I_{3} & O_{3} \\
O_{3} & T\left(\varphi_{b}\right)
\end{array}\right]
$$

where $T\left(\varphi_{b}\right)$ can be given by

$$
T\left(\varphi_{b}\right)=\left[\begin{array}{ccc}
0 & -s \psi & c \psi c \theta \\
0 & c \psi & s \psi c \theta \\
1 & 0 & -s \theta
\end{array}\right]
$$

The state vector $x_{b}$ can be rewritten as follows

$$
x_{b}=\left[\begin{array}{l}
\mu_{b} \\
\sigma_{b}
\end{array}\right], \mu_{b}=\left[\begin{array}{c}
p_{b} \\
\psi
\end{array}\right], \sigma_{b}=\left[\begin{array}{l}
\theta \\
\phi
\end{array}\right] .
$$

The differential kinematics expression $v_{e}$ can be written as [17]

$$
v_{e}=J_{\gamma}\left(\sigma_{b}, \gamma\right) \dot{\gamma}+J_{\sigma}\left(\sigma_{b}, \gamma\right) \dot{\sigma}_{b}
$$

where $\gamma=\left[\begin{array}{ll}\mu_{b}^{T} & q^{T}\end{array}\right]$ is the vector of controlled variables, $J_{\gamma}$ is composed by first 4 columns of $J_{b} T_{A}\left(\varphi_{b}\right), J_{\sigma}$ is composed by the last 2 columns of $J_{b} T_{A}\left(\varphi_{b}\right), J_{\sigma}$ and $J_{\gamma}=\left[\begin{array}{ll}J_{\gamma} & J_{e b}\end{array}\right]$.
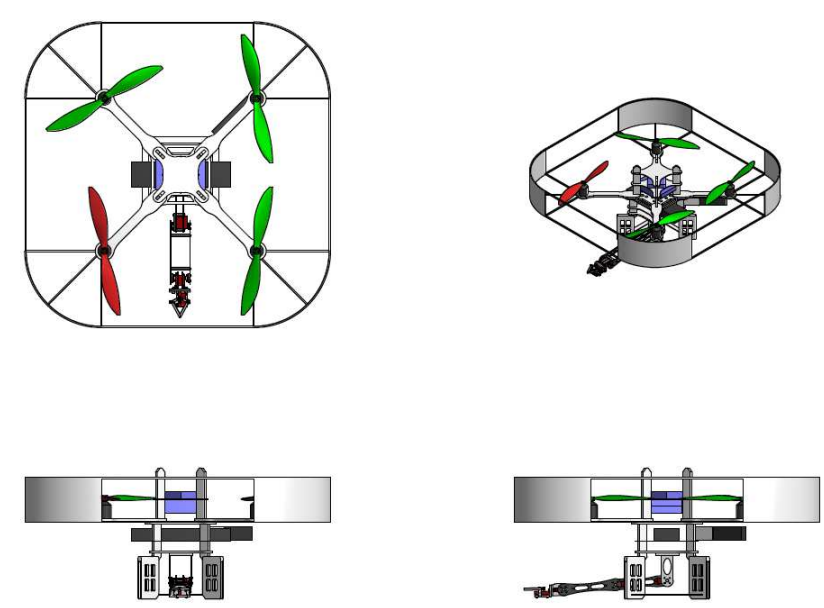

Fig. 1: Aerial Manipulation Vehicle.

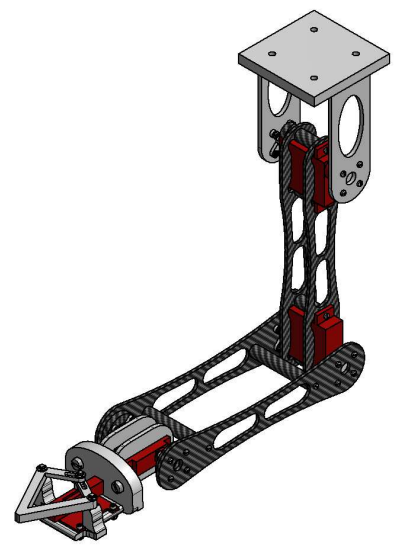

Fig. 2: 2 DOF Manipulator with base and two links. Joint1 and Joint-2 placed at the end of the base and link-1 respectively.

\section{B. Dynamics}

The dynamic model of the aerial manipulation vehicle can be given using Euler-Lagrange method as follows [17]

$$
M(\chi) \ddot{\chi}+C(\chi, \dot{\chi}) \dot{\chi}+g(\chi)=u
$$




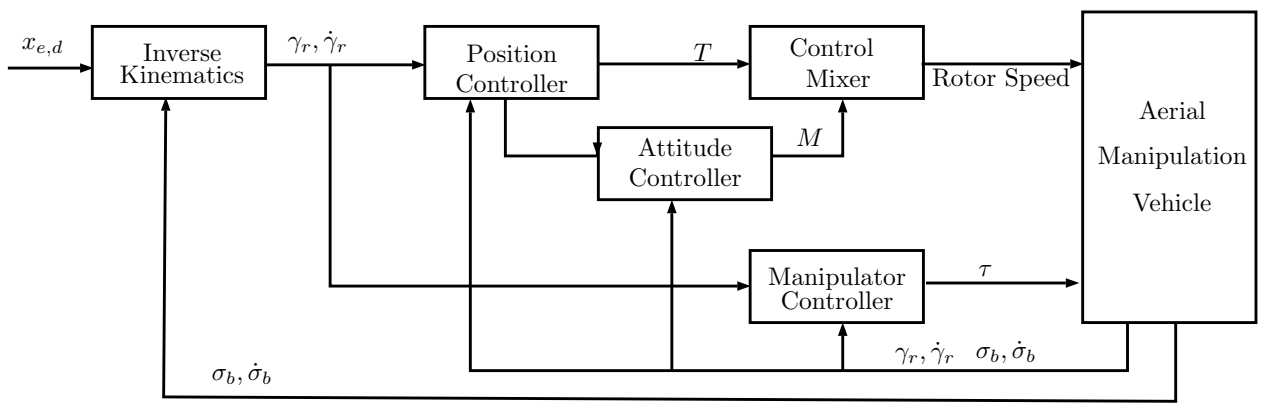

Fig. 3: Aerial Manipulation Vehicle: Control Structure.

where $\chi=\left[\begin{array}{cc}x_{b}^{T} & q^{T}\end{array}\right], M$ is the symmetric and positive definite inertia matrix, $C$ includes the coriolis and centrifugal quantities, $g$ is the gravity term and $u$ is the input vector [17]

$$
u=\left[\begin{array}{c}
u_{f} \\
u_{\nu} \\
u_{\tau}
\end{array}\right]=\left[\begin{array}{c}
R_{b}(\varphi) f_{b}^{b} \\
T^{T}\left(\varphi_{b}\right) R\left(\varphi_{b}\right) \nu_{b}^{b} \\
\tau
\end{array}\right]
$$

where $\tau$ are the torques applied to the manipulator joint, $f_{b}^{b}$ and $\nu_{b}^{b}$ are the forces and moments on the quadrotor expressed in $\mathcal{B}$.

\section{CONTROL}

The structure of the control scheme can be seen in Figure-3. The outer layer consist of the Closed Loop Inverse Kinematics followed by position and attitude control for the quadrotor and joint control for the manipulator. Here we will briefly discuss the control schemes involved.

\section{A. Closed Loop Inverse Kinematics}

The purpose of the Closed Loop Inverse Kinematics algorithm is to generate references to the position, attitude and joint control loops for the quadrotor and manipulator respectively.The closed loop inverse kinematics is given by [17]

$$
\begin{aligned}
\dot{\gamma}_{r}= & J_{\gamma}^{\dagger}\left(\sigma_{b}, \gamma_{r}\right) T_{A}\left(\varphi_{e, r}\right)\left(\dot{x}_{e, d}+K e\right) \\
& J_{\gamma}^{\dagger}\left(\sigma_{b}, \gamma_{r}\right) J_{\sigma}\left(\sigma_{b}, \gamma_{r}\right) \dot{\sigma}_{b}
\end{aligned}
$$

where $J_{\gamma}^{\dagger}$ is the pseudoinverse of $J_{\gamma}, K$ is the symmetric positive definite gain and $e=x_{e, d}-x_{e, r}$ is the error of the end effector position and attitude.

\section{B. Closed Loop Control Structure}

The basic hierarchical control structure of the quadrotor can be seen in Figure-3. The quadrotor control has inner attitude and outer position loops. A simple controller for the roll and pitch channels could then be

$$
U_{r p}(t)=K_{p}\left[\varepsilon_{r p}(t)-K_{d} \dot{\Theta}_{r p}(t)\right]
$$

where we have $\varepsilon_{r p}=\Theta_{r p}^{*}-\Theta_{r p}$, with $\Theta_{r p}^{*}$ as the roll and pitch demand, $U_{r p}$ gives us pitch $\left(\tau_{y}\right)$ and roll torque $\left(\tau_{x}\right)$. A PID control is used for the yaw control loop

$$
U_{\text {yaw }}(t)=K_{p}\left[\varepsilon_{\text {yaw }}(t)+K_{i} \int_{0}^{t} \varepsilon_{\text {yaw }}(\delta) d \delta-K_{d} \dot{\Omega}_{z}(t)\right]
$$

where $\varepsilon_{y a w}=\psi^{*}-\psi$ is the yaw error with yaw demand $\psi^{*}$.

The outer-loop consists of a $x, y$ position control with PID type structure, the output of which is the pitch and roll demand

$$
\begin{aligned}
\Theta_{r p}^{*}(t)=K_{p}\left[\varepsilon_{x y}(t)+K_{i} \int_{0}^{t} \varepsilon_{x y}(\delta) d \delta\right. & -K_{d} v_{x y}(t) \\
& \left.-K_{d d} \dot{v}_{x y}(t)\right]
\end{aligned}
$$

where $\varepsilon_{x y}=\xi_{x y}^{*}-\xi_{x y}$ is the horizontal position error and $v_{x y}$ is the position rate and $\dot{v}_{x y}$ is the respective acceleration. For the height control an independent PID control

$$
T(t)=K_{p}\left[\varepsilon_{z}(t)+K_{i} \int_{0}^{t} \varepsilon_{z}(\delta) d \delta-K_{d} v_{z}(t)-K_{d d} \dot{v}_{z}(t)\right]
$$

is used where $\varepsilon_{z}=\xi_{z}^{*}-\xi_{z}$ is the $z$ position error and $v_{z}$ is the $z$ position rate and $\dot{v}_{z}$ is the respective acceleration.

For the manipulator control problem an independent joint control method was used preferable with PD configuration such as

$$
U_{m}(t)=K_{p}\left[K_{p 1} \tilde{q}(t)-K_{d} \dot{q}(t)\right]
$$

where $\tilde{q}$ is a joint angle error.

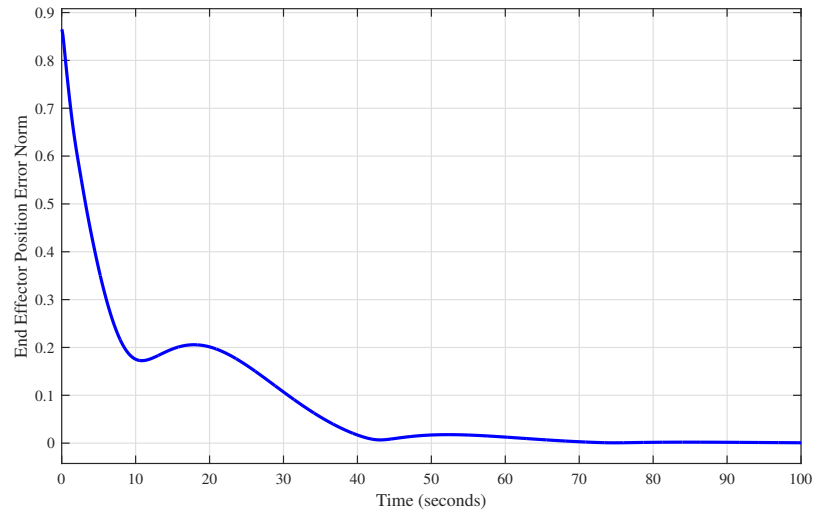

Fig. 4: Position Control: error norm .

\section{Simulation}

The above discussed algorithms are applied to the nonlinear simulation model of Asctec Pelican Quadrotor equipped with 


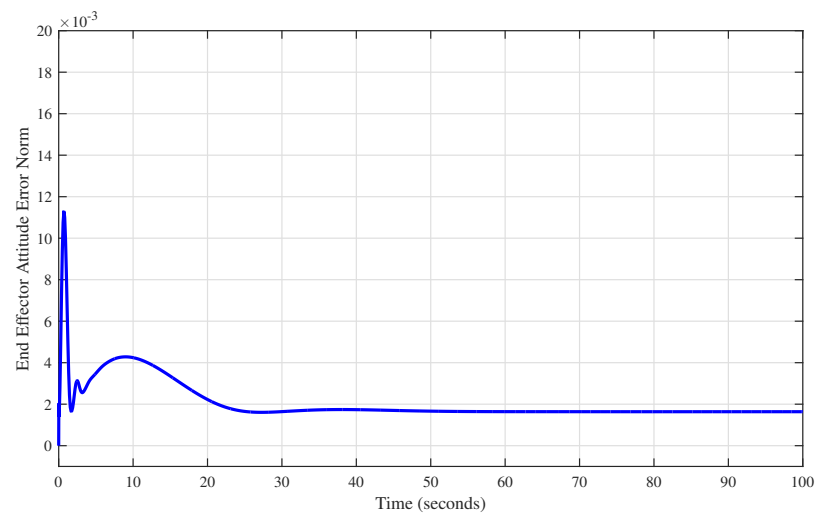

Fig. 5: Position Control: Attitude error norm .

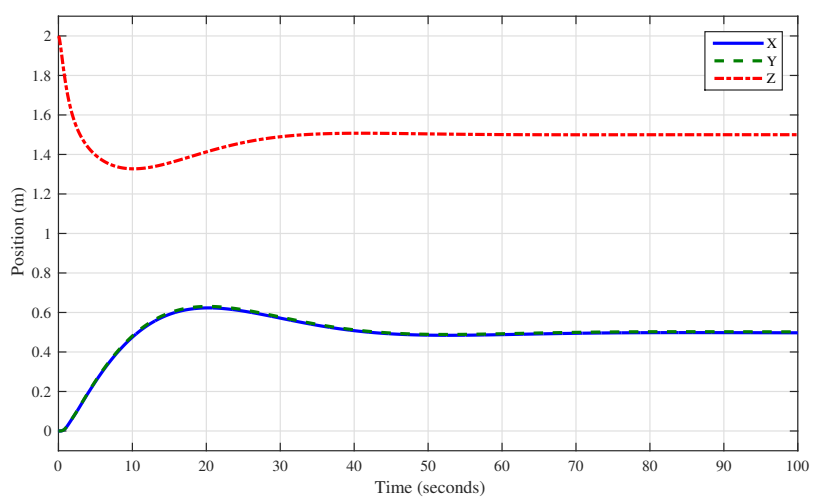

Fig. 6: Position Control: Quadrotor Position .

a 2-DOF manipulator. The Asctec Pelican is a quadrotor with mass $2 \mathrm{Kg}$ and inertia $I=\operatorname{diag}\left(\left[\begin{array}{lll}1.24 & 1.24 & 2.48\end{array}\right]\right.$. The model parameters have been obtained from [17]. The quadrotor is equipped with a 2 degree of freedom robotic arm with a base and 2 links. The design of the light weight manipulator can be seen in Figure-2. The total mass of the robotic arm is $110 \mathrm{~g}$. The complete Aerial Manipulator system was modelled in CAD and imported into Simulink-

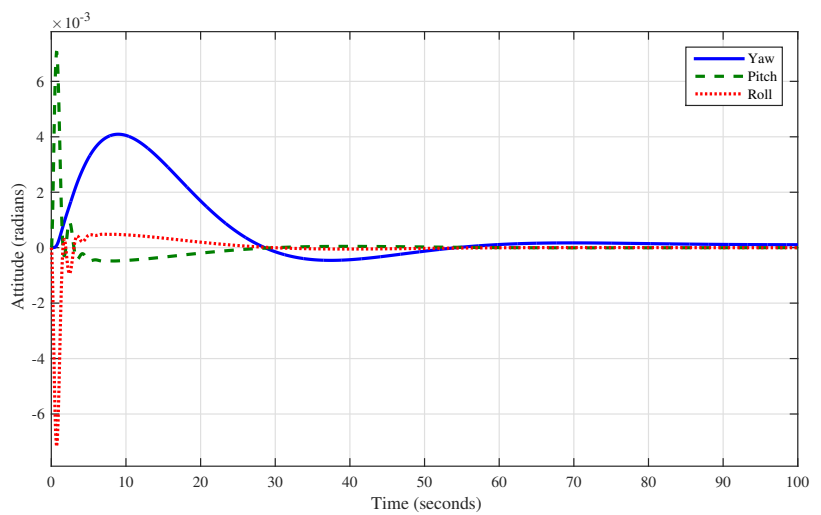

Fig. 7: Position Control: Quadrotor Attitude .

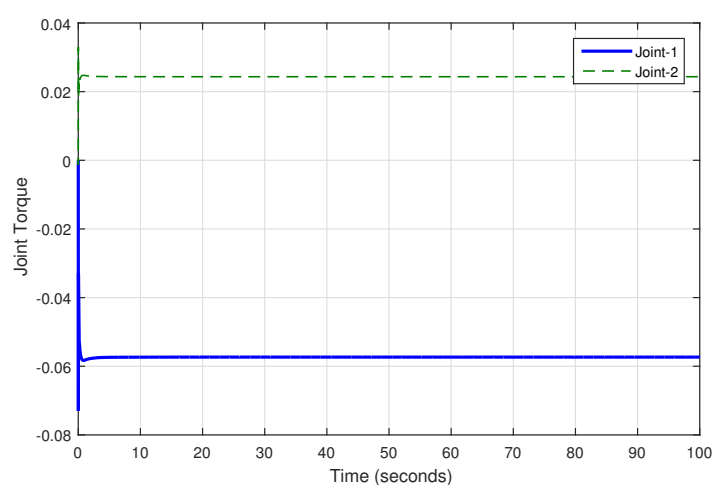

Fig. 8: Position Control: Manipulator Joint Torque.

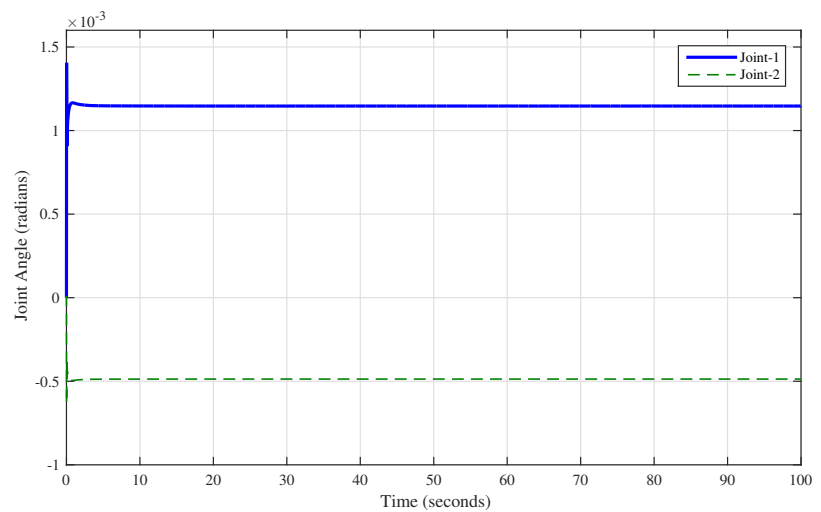

Fig. 9: Position Control: Manipulator Joint Angle.

Simmechanics environment which finally serves as a high fidelity nonlinear simulator.

In order to test the controller the key task here is position control in operation space. That is the end-effector has to reach a final position starting from an initial position. The Aerial Manipulator starts from a hovering position at $\{0+0.27587,0,2-0.1184\}$ with manipulator joint angle $[0,0]$ and has to reach $\{0.5+0.27587,0.5,1.5-0.1184\}$.

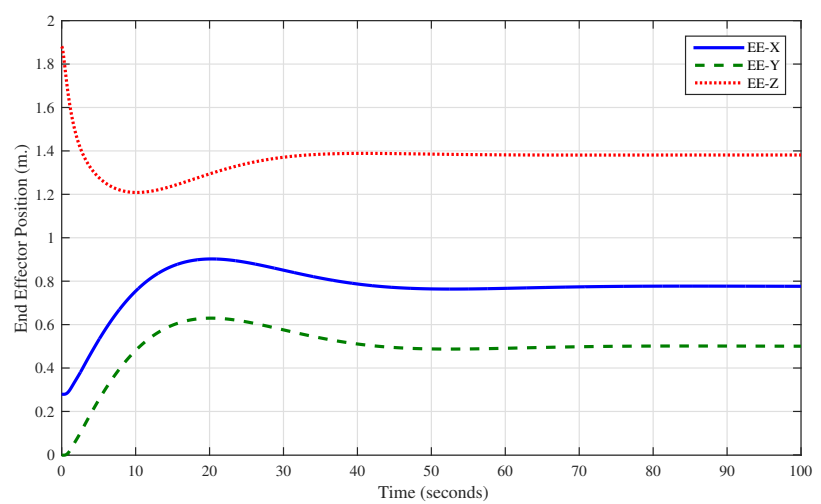

Fig. 10: Position Control: Manipulator Position. 


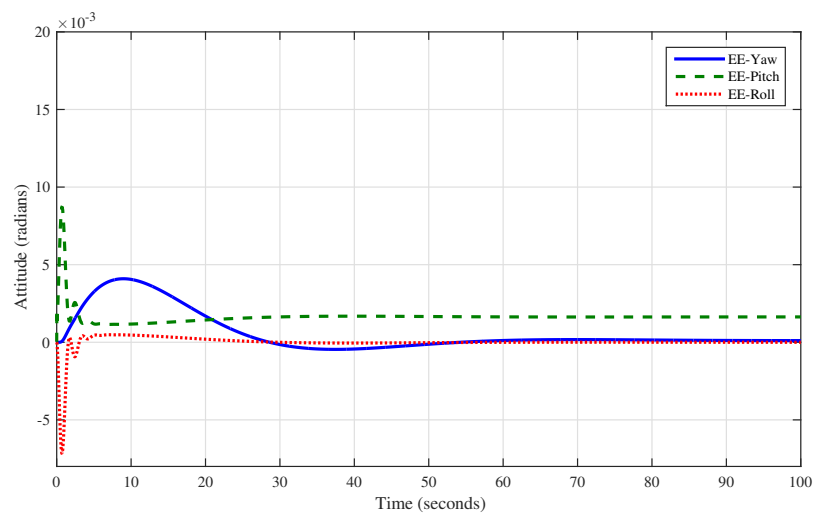

Fig. 11: Position Control: Manipulator Attitude.

During this simulation a single set of controller parameters are used. The inner attitude control has the controller gains $K_{p}=5, K_{d}=5$ for roll and pitch control where as the yaw control gains are $K_{p}=-3, K_{i}=0.001, K d=0.01$. Similarly the position control has the following controller gains: $K_{p}=[10,-10], K_{i}=[0.1,0.1], K_{d}=[7,7], K_{d d}=$ $[1,1]$ for horizontal position $(x-y$ axis) control while for vertical position (z-axis) control the gains were $K_{p}=10, K_{i}=$ $0.1, K_{d}=5, K_{d d}=1$. The manipulator controller gains are $K_{p}=1, K_{p 1}=50, K_{d}=0.1$ for Joint-1 and Joint-2 and the the inverse kinematics gains are $K=[1,1,1,1,1,1]$. It must be brought to notice that the above controller gains were chosen by a tedious trial and error method in order to achieve the design goals. At this stage no effort has been taken to optimize the controller gains.

The End-Effector position error norm and attitude error norm can be seen in Figure-4 and Figure-5 respectively. Similarly the position and attitude of the quadrotor can be seen in Figure-6 and Figure-7 respectively. Considering the manipulator control in Figure-8 and Figure- 9 we can see the joint torques applied to control and the joint angles respectively. In Figure-10 and Figure-11 we can see the position and attitude of the manipulator End-Effector. From the above figures we can see that end-effector position error norm converges to $0 \mathrm{~m}$ while the attitude converges to $2 \times 10^{-3}$ radians. We can also clearly observe that the position and attitude states of the quadrotor and manipulator reaches steady states.

\section{CONClusion}

In the current paper we have successfully discussed the problem of position control of Aerial Manipulation Vehicle in the operational space. The kinematics and dynamics of the aerial manipulator was discussed, the hierarchical control structure was addressed and finally simulation studies were performed using a nonlinear model. The future perspectives of this research include detailled stability analysis and control design to perform more advanced tasks.

\section{REFERENCES}

[1] P. E. I. Pounds, D. R. Bersak, and A. M. Dollar, "Grasping from the air: Hovering capture and load stability," in 2011 International conference on Robotics and Automation, 2011, pp. 2491-2498.

[2] L. Marconi, R. Naldi, and L. Gentili, "Modelling and control of a flying robot interacting with the environment," Automatica, vol. 47, pp. 2571$2583,2011$.

[3] V. Lipiello and F. Ruggiero, "Cartesian impedence control of a uav with a robotic arm," in 10th IFAC Symposium on Robot Control, 2012.

[4] — "Exploiting redundancy in cartesian impedance control of UAVs equipped with a robotic arm," in 2012 IEEE/RSJ International Conference on Intelligent Robots and Systems, 2012, pp. 3768-3773.

[5] M. Orsag, C. Korpela, and P. Oh, "Modeling and control of mm-uav: Mobile manipulating unmanned aerial vehicle," Journal of Intell Robot Sys, vol. 69, pp. 227-240, 2013.

[6] A. Khalifa, M. Fanni, A. Ramadan, and A. Abo-Ismail, "Modeling and control of a new quadrotor manipulation system," in 2012 First International Conference on Innovative Engineering Systems (ICIES), 2012, pp. 109-114.

[7] M. Orsag, C. Korpela, S. Bogdan, and P. Oh, "Lyapunov based model reference adaptive control for aerial manipulation," in 2013 International Conference on Unmanned Aircraft Systems (ICUAS), may 2013, pp. 966-973.

[8] K. Kondak, K. Kreiger, A. Albu-Schaeffer, M. Schwarzbach, M. Laiacker, I. Maza, A. Rodriguez-Castano, and A. Ollero, "Closed-loop behaviour of an autonomous helicopter equipped with a robotic arm for aerial manipulation tasks," International Journal of Advanced Robotic Systems, vol. 10, 2013.

[9] V. Ghadiok, J. Goldin, and W. Ren, "Autonomous indoor aerial gripping using a quadrotor," in 2011 IEEE/RSJ International Conference on Intelligent Robots and Systems, september 2011, pp. 4645-4651.

[10] I. Palunko, P. Cruz, and R. Fierro, "Agile load transportation. safe and efficient load manipulation with aerial robots," Robotics and Automation Magazine, pp. 69-79, 2012.

[11] G. Antonelli, F. Arrichiello, S. Chiaverini, and P. R. Giordano, "Adaptive trajectory tracking for quadrotor mavs in presence of uncertainities and external disturbances," in 2013 IEEE/ASME International conference on Advanced Intelligent Mechatronics (AIM), July 2013, pp. 1337-1342.

[12] G. Antonelli, E. Cataldi, P. R. Giordano, S. Chiaverini, and A. Franchi, "Experimental validation of a new adaptive control scheme for quadrotors mavs," in 2013 IEEE/RSJ International conference on Intelligent Robots and Systems (IROS), November 2013, pp. 2439-2444.

[13] S. Kannan, M. Alma, M. A. Olivares-Mendez, and H. Voos, "Adaptive control of aerial manipulation vehicle," in Control System, Computing and Engineering (ICCSCE), 2014 IEEE International Conference on, Nov 2014, pp. 273-278.

[14] S. Kannan, M. A. Olivares-Mendez, and H. Voos, "Modeling and control of aerial manipulation vehicle with visual sensor," in 2nd IFAC Workshop on Research, Education and Development of Unmanned Aerial Systems, vol. 2, November 2013.

[15] V. Lippiello, J. Cacace, A. Santamaria-Navarro, J. Andrade-Cetto, M. . Trujillo, Y. R. Esteves, and A. Viguria, "Hybrid visual servoing with hierarchical task composition for aerial manipulation," IEEE Robotics and Automation Letters, vol. 1, no. 1, pp. 259-266, Jan 2016.

[16] S. Kim, S. Choi, and H. J. Kim, "Aerial manipulation using a quadrotor with a two dof robotic arm," in 2013 IEEE/RSJ International Conference on Intelligent Robots and Systems, Nov 2013, pp. 4990-4995.

[17] G. Arleo, F. Caccavale, G. Muscio, and F. Pierri, "Control of quadrotor aerial vehicles equipped with a robotic arm," in Control Automation (MED), 2013 21st Mediterranean Conference on, June 2013, pp. 11741180.

[18] F. Caccavale, G. Giglio, G. Muscio, and F. Pierri, "Adaptive control for uavs equipped with a robotic arm," in 19th IFAC World COngress, vol. 19, August 2014, pp. 11 049-11 054. 